\title{
Breast cancer quality of life evaluation in Mexican Women at La Raza Hospital, Mexico City:A preliminary approach
}

\author{
Jacobo Alejandro Gómez- \\ Rico' \\ Marina Altagracia-Martínez' \\ Jaime Kravzov-Jinich' \\ Rosario Cárdenas-Elizalde \\ Juan Carlos Hinojosa-Cruz ${ }^{2}$ \\ Consuelo Rubio-Poo ${ }^{3}$ \\ 'Departments of Biological Systems \\ and Healthcare, Biological and Health \\ Sciences Division (DCBS), Universidad \\ Autónoma Metropolitana-Xochimilco \\ (UAM-X), Xochimilco, Mexico; ${ }^{2}$ La \\ Raza Hospital of the Mexican Social \\ Security Institute (IMSS), Mexico \\ City, Mexico; ${ }^{3}$ Universidad Nacional \\ Autónoma de México (UNAM), \\ Faculty of Professional Studies, \\ Zaragoza (FES-Zaragoza), Mexico
}

Correspondence: Marina AltagraciaMartínez

Calle La Joya \#I7 casa 5, Colonia San Juan Tepepan, México DF, CP I6020, Delegación Xochimilco, Mexico Tel +52 555483 7250/54837098 $\mathrm{Fax}+525556060535$ Email altagracia |4200@lycos.com

\begin{abstract}
Breast cancer (BC) is the second leading cause of death among Mexican women over 40 years of age. This study aimed to identify and examine the effects of cancer stage and surgical treatment on the quality of life (QOL) of Mexican women with early stage breast cancer (ESBC) treated with either modified radical mastectomy (MRM) or breast conservative surgery (BCS), plus adjuvant chemotherapy. The QLQ-C30 and QLQ BR-23 questionnaires were used to assess QOL. Sociodemographic characteristics and clinical factors of 102 women with early BC were also evaluated; analysis of variance (ANOVA) was performed and a statistical significance of $p<0.05$ was assumed. Most women were of reproductive age. Meaningful differences in QOL as a result of surgical treatment, in women receiving BCS compared with those receiving MRM, were limited to body image. We conclude that MRM and BCS are essentially equivalent choices in terms of QOL, with the exception of the impact on body image. In general, women who received BCS had a better perceived QOL.
\end{abstract}

Keywords: quality of life, breast cancer, Mexican women

\section{Introduction}

Breast cancer (BC) is the leading cause of cancer death among women around the world. ${ }^{1,2} \mathrm{BC}$ and cervicouterine cancer are the most common cancers in Mexican women. ${ }^{3} \mathrm{BC}$ is the second leading cause of death among Mexican women aged 40 or more, killing approximately 10 women daily; an average of one death every two and a half hours. ${ }^{4}$

The first step in combatting cancer is making the diagnosis. This requires a combination of careful clinical evaluation and diagnostic tests, which may include endoscopy, imaging, histopathology, cytology, and laboratory tests. ${ }^{3}$ Once the diagnosis is confirmed, the cancer is staged. A decision on therapy is made and an experimental treatment protocol established. ${ }^{5}$

For the majority of patients diagnosed with early stage breast cancer (ESBC), the options for local treatment are mastectomy or breast conservative surgery (BCS) with radiotherapy. ${ }^{6,7}$

The objectives of $\mathrm{BC}$ treatment are eradication of the cancer, prolongation of life, and improved quality of life. The diagnosis of breast cancer and its subsequent treatment have been shown to have a significant influence on a woman's physical functioning, mental health, and well-being, causing substantial disruption to her quality of life (QOL). ${ }^{1,6,8,9}$

A feature common to all forms of cancer is the failure of the mechanisms which normally regulate cell growth, proliferation, and death. The resulting tumor, which begins as a small anomaly, becomes larger and more injurious, invades neighboring cells, and is eventually propagated in other parts of the body. ${ }^{10}$ 
Measures of health-related quality of life (HRQOL) are becoming increasingly important in assessing the effects of chronic illness, and the medical interventions designed to treat them. National spending on medical care is growing rapidly in every country. ${ }^{2,11}$ HRQOL measures could enhance our understanding of health status in any given country, and promote further study of the economic causes and effects of changes in health status. ${ }^{2}$

HRQOL is a multidimensional construct that represents the patient's perception of the effects of an illness and its associated therapy on his or her day-to-day functioning. ${ }^{12}$ Once active treatment is completed, some women celebrate; others become anxious about recurrence and feel that they have lost a proactive shield against cancer. ${ }^{13}$ Many studies report persistent disturbances in family functioning because marital issues were ignored during the cancer treatment phase. Body image and age, especially after mastectomy, have also been detected as concerns. ${ }^{14,15}$

In Mexico, very little (if any) research has compared the HRQOL of women treated with modified radical mastectomy (MRM), with the HRQOL of women experiencing BCS. Factors such as patient education, spouse support and employment status, financial stability, and disease stage, have been found to influence not only quantity of survival, but also HRQOL. ${ }^{1}$ To enhance the QOL for Mexican women with BC, more QOL studies to investigate the effect of sociodemographic and clinical factors on their QOL are needed.

The objectives of the present study were to identify and examine the relationship between cancer stage and surgical treatment with the QOL of two groups of Mexican women with ESBC, who had been treated with MRM or BCS plus adjuvant chemotherapy at La Raza, a large public hospital in Mexico City with specialized breast cancer services.

\section{Materials and methods Study design and sample selection}

A prospective, descriptive and analytical study was conducted. The study population consisted of women diagnosed with ESBC (stages I or II). Mexican epidemiological sources show that most women diagnosed with BC are older than 35 years. ${ }^{3,10}$ Consequently, eligibility criteria included: a) age $>35$ years and $<65$ years; b) a primary diagnosis of $\mathrm{BC}$ treated with a surgical procedure (BCS or MRM); c) an existing medical record at La Raza hospital before the start of the study, and attending this hospital as an outpatient for chemotherapy treatment during the study period; and d) capable of completing the European Organization for Research and Treatment of Cancer Quality of Life Questionnaire version 3
(EORT QLQ-C30), and the Breast Cancer-Specific Quality of Life Questionnaire (QLQ BR-23). ${ }^{16,17}$ Both QOL questionnaires were translated into Spanish and validated in Mexico by the EORT Quality of Life Group and have been tested for validity in various populations. ${ }^{18}$ The EORT Quality of Life Group granted written permission to the first author of the study.

Participants provided written consent, and approval was granted by the La Raza Hospital Health Service Research and Ethics Committee.

The initial sample screening was completed with all the medical records of women with cancer in the study period. Most women in the preliminary sample (506) did not meet the inclusion criteria, that is, they were older than 35 years, had an advanced stage of BC (III and IV), or/were suffering from other types of cancer. Consequently, only 102 women of a total of 506 women with medical records were included in the present study. The questionnaires were applied only once during the study period (which lasted from September to November 2007).

\section{Data collection and study measures}

Information about treatment and clinical factors was obtained from patient medical records. BC stage was classified using the American Joint Committee on Cancer (AJCC) TNM Staging System for Breast Cancer. A 17-item questionnaire was applied to the study population sample (102 women with BC in stages I-II). Questionnaire items included sociodemographic characteristics (age, level of education, marital status, employment status) and clinical factors (breast cancer stage and treatment). Clinical factor responses were confirmed from patient medical records. QOL was measured by self-completed questionnaires.

\section{Quality of life questionnaire}

QOL was assessed by patient responses to a generic cancer instrument, the QLQ-C30 and QLQ BR-23, as mentioned above.

\section{Scoring of QLQ-C30 and QLQ-BR23}

The QLQ-C30 contains 30 questions and has been grouped by the instrument's authors into three scales: one global QOL scale (two items), five function scales (15 items) and nine symptom scales (13 items). Scale scores are the average of the component item responses, transformed linearly to a range of 0-100 (as described in the User's Guide). ${ }^{19}$ The QLQ-BR23 comprises 23 questions and has been grouped by the instrument's author into two scales: four function 
scales (13 items) and four symptom scales (eight items). Scale scores are transformed linearly to a range of 0-100. For both QOL questionnaires (QLQ-C30 and QLQ-BR23) the function scales and the global QOL scale, a higher score corresponds to a better QOL. For the symptom scales, a higher score indicates more frequent and/or intense symptom experience and thus a lower QOL. ${ }^{20}$

\section{Statistical analysis}

Each QOL measure was standardized to a score ranging from 0 to 100 by using the linear transformation in the EORTC QLQ Scoring Manual. Bivariate associations between stage and treatment and each of the QOL measures were investigated using analysis of variance (ANOVA). A statistical significance of $\mathrm{p}<0.05$ was assumed. All analyses were performed using SPSS version 13 (SPSS Inc., Chicago, IL).

\section{Results}

Table 1 shows the study population categorized by sample characteristics and by primary surgical treatment. 102 women with BC in stages I-II were included; 44 (43.1\%) subjects were aged 56 to $65 ; 32$ (31.4\%) subjects were aged 46 to 55 . Most respondents, that is, $60(58.8 \%)$, had completed elementary school, 24 (23.5\%) middle school, 13 (12.74\%) technical studies and only $5(4.9 \%)$ had reached a post-secondary degree. 65 (63.4\%) were homemakers and 23 (22.54\%) were formally employed. Three (2.94\%) were self-employed (Table 1$)$. Ninety-three subjects $(91.17 \%)$ were in stage II and $9(8.82 \%)$ were in stage I (Table 1$)$.

Eighty-four (82.4\%) women received MRM and only $18(17.6 \%)$ BCS. In relation to treatments (chemotherapy), $86(84.31 \%)$ women who had MRM were treated with 5-fluorouracil (5-FU), epirubicin, and cyclophosphamide (FEC). Of the BCS patients, 15 (14.6\%) were treated with cyclophosphamide, methotrexate, 5-fluorouracil (CMF), and one $(0.98 \%)$ with other treatment agents. All subjects completed six cycles of chemotherapy during the study period (Table 1).

Sixty-eight (66.7\%) women were currently married or had a domestic partner and 34 (33.3\%) were single.

Table I Sample characteristics of the study population overall and by primary surgical treatment $(\mathrm{N}=102)$

\begin{tabular}{|c|c|c|c|c|c|}
\hline Characteristics & N 102 & Overall (\%) & MRM N = 84 (\%) & $\begin{array}{l}\text { Breast-conserving } \\
\text { surgery } N=18(\%)\end{array}$ & $\begin{array}{l}\text { p-value } \\
\text { (ANOVA) }\end{array}$ \\
\hline \multicolumn{6}{|l|}{ Sociodemographic } \\
\hline \multicolumn{6}{|l|}{ Age } \\
\hline$>35<45$ & 26 & 25.5 & 23.1 & 38.8 & 0.272 \\
\hline$>46<55$ & 32 & 31.4 & 30.4 & 38.8 & 0.392 \\
\hline$>56>65$ & 44 & 43.1 & 48.7 & 22.2 & $0.026^{*}$ \\
\hline \multicolumn{6}{|l|}{ Education level } \\
\hline Elementary school & 60 & 58.8 & 60.7 & 50 & $0.03 I^{*}$ \\
\hline Junior high & 24 & 23.5 & 23.8 & 22.2 & 0.342 \\
\hline Technical & 13 & 12.74 & 13.09 & II.I & 0.605 \\
\hline Bachelor degree & 5 & 4.9 & 2.38 & 16.6 & 0.571 \\
\hline \multicolumn{6}{|l|}{ Occupation } \\
\hline Unemployed & II & 10.78 & 8.3 & 22.2 & $0.043^{*}$ \\
\hline Housekeeper & 65 & 63.72 & 67.8 & 44.4 & $0.037^{*}$ \\
\hline Employee & 23 & 22.54 & 22.6 & 22.2 & 0.272 \\
\hline Self-employed & 3 & 2.94 & 1.19 & 11.1 & 0.041 \\
\hline \multicolumn{6}{|l|}{ Clinical factors } \\
\hline Stage I & 9 & 8.82 & 3.65 & 33.3 & $0.036 *$ \\
\hline Stage II & 93 & 91.17 & 96.34 & 66.6 & $0.021 *$ \\
\hline \multicolumn{6}{|l|}{ Treatment } \\
\hline FEC & 86 & 84.31 & 84.5 & 83.3 & 0.562 \\
\hline CMF & 15 & 14.70 & 14.2 & 16.6 & 0.813 \\
\hline Others & I & 0.98 & 1.19 & 0 & 0.260 \\
\hline
\end{tabular}

Note: *P $<0.05$ statistical significance.

Abbreviations: ANOVA, analysis of variance; CMF, cyclophosphamide, methotrexate, 5-fluorouracil; FEC, 5-fluorouracil, epirubicin, and cyclophosphamide; MRM, modified radical mastectomy. 
Tables 2 and 3 show the results of the QOL questionnaires. As mentioned in the methodological section (scoring QLQ-C30 and QLQ-BR23), for both QOL questionnaires (QLQ-C30 and QLQ-BR23), the function scales and the global QOL scale, a higher score corresponds to a better QOL; for the symptom scales, a higher score indicates more frequent and/or intense symptom experience and thus a lower QOL. ${ }^{20}$

Table 2 shows the QLQ-C30 results. No significant difference in global state of health was observed between the two groups $(\mathrm{p}>0.05)$. Physical function was significantly different between women treated with MRM and women treated with conservative surgery $(\mathrm{p}<0.05)$. On the symptom scale, the items with significant differences between the two groups were nausea and vomiting $(\mathrm{p}<0.05)$.

Table 2 also shows that women who had conservative surgery had a higher level than women with MRM in three out of five of the function scales. The two exceptions were emotional function (72.22 vs 66.20) and cognitive function (87.10 vs 78.70).

Table 3 shows the results of the BC-specific QLQ-BR23 questionnaire by type of surgery. As a result of the variance analysis (ANOVA), a significant difference can be seen between the MRM group and the BCS group on the function, body image, and future perspective scales $(p<0.05)$. The conservative surgery group scored considerably higher on health and function (87.03 vs 19.41 and 72.22 vs 29.76, respectively). The results show a better QOL for the BCS group than for the MRM accordingly to the scoring method already described.

All items on the symptom scale were significantly different in the two groups except for concern about hair loss $(\mathrm{p}>0.05)$.

\section{Discussion}

The age distributions of both groups (MRM and conservative surgery) are consistent with those found in other countries. ${ }^{12,21}$

Women who had completed only elementary school received MRM, suggesting that education plays an important role in the early detection of BC. ${ }^{6,12,21}$ A lower level of education may also be related to patient willingness to follow physician suggestions to have MRM, rather than request BCS.

Most of the women were homemakers or unemployed. Our results differ from those of published studies from other geographical areas, where most subjects were employed. ${ }^{6,12,21}$

The results of the present investigation provide insights on the effects of early stage breast cancer and its treatment on QOL. These findings support those of studies conducted in other populations which suggest that patient physicalpsychological concerns, such as insomnia and lack of appetite, are similar regardless of the stage or type of surgery. ${ }^{6,12,21}$

Table 2 Results of the QLQ-C30 questionnaire by primary surgery

\begin{tabular}{|c|c|c|c|c|}
\hline Name & Scale & MRM N $=84$ & $\operatorname{BCS} N=18$ & ANOVA $p$ value \\
\hline Global health status & QL & 67.65 & 72.22 & 0.181 \\
\hline \multicolumn{5}{|l|}{ Functional scales } \\
\hline Physical functioning & PF & 86.34 & 91.85 & $0.036 *$ \\
\hline Role functioning & RF & 72.22 & 76.85 & 0.312 \\
\hline Emotional functioning & $\mathrm{EF}$ & 72.22 & 66.20 & 0.260 \\
\hline Cognitive functioning & $\mathrm{CF}$ & 87.10 & 78.70 & 0.073 \\
\hline Social functioning & SF & 79.56 & 79.63 & 0.991 \\
\hline \multicolumn{5}{|l|}{ Symptom scales } \\
\hline Fatigue & FA & 25.26 & 22.83 & 0.577 \\
\hline Nausea and vomiting & NV & 17.06 & 6.48 & $0.043^{*}$ \\
\hline Pain & PA & 24.99 & 16.66 & 0.056 \\
\hline Dyspnea & DY & 9.52 & 7.40 & 0.605 \\
\hline Insomnia & SL & 15.07 & 20.36 & 0.365 \\
\hline Appetite loss & AP & $10.7 \mid$ & 9.25 & 0.805 \\
\hline Constipation & $\mathrm{CO}$ & 13.49 & 16.66 & 0.557 \\
\hline Diarrhea & DI & 4.36 & 7.4 & 0.392 \\
\hline Financial difficulties & $\mathrm{FI}$ & 39.28 & 27.77 & 0.128 \\
\hline
\end{tabular}

Note: *P $<0.05$ statistical significance.

Abbreviations: ANOVA, analysis of variance; BCS, breast cancer surgery; MRM, modified radical mastectomy; QLQ-C30, European Organization for Research and Treatment of Cancer Quality of Life Questionnaire version 3. 
Table 3 Results of the QLQ-BR23 questionnaire by primary surgery

\begin{tabular}{lllll}
\hline Name & Scale & MRM N = 84 & BCS N= I8 & ANOVA P value \\
\hline Functional scales & & & & $0.0285^{*}$ \\
Body image & BRI & 19.41 & 87.03 & $0.037 I^{*}$ \\
$\begin{array}{l}\text { Future perspective } \\
\text { Symptom scales }\end{array}$ & BRFU & 29.76 & 72.22 & $0.0133^{*}$ \\
$\begin{array}{l}\text { Systemic therapy } \\
\text { side effects }\end{array}$ & BEST & 40.93 & 28.04 & $0.00 I^{*}$ \\
Breast symptoms & & & 8.79 & $0.009^{*}$ \\
Arm symptoms & BROS & 54.76 & 9.87 & 0.278 \\
Upset by hair loss & BRAS & 44.44 & 90.74 & \\
\hline
\end{tabular}

Note: *P $<0.05$ statistical significance.

Abbreviations: ANOVA, analysis of variance; BCS, breast cancer surgery; MRM, modified radical mastectomy; QLQ BR-23, Breast Cancer-Specific Quality of Life Questionnaire.

The results of the QLQ-C30 questionnaire by type of surgery (Table 2) show that the majority of parameters assessed (excepting physical function, and nausea and vomiting) were not significantly different between women treated with MRM and women treated with conservative surgery. Women in the BCS group showed higher values on the global health scale (72.22), indicating a relatively better QOL, than those who received MRM (67.65).

The results may indicate that mastectomy does not itself substantially reduce QOL, compared with conservative surgery, apart from nausea and vomiting $(17.06$ versus 6.48 in women treated with BCS $)(\mathrm{p}<0.05)$. $^{6,12}$

The BCS group showed higher values on the function scales than the MRM group in all dimensions except emotional function (72.22 vs 66.20).

As noted above, the only significant result when comparing symptoms between the two groups was nausea and vomiting $(p<0.05)$. The symptom scale indicates the health problems experienced by the patients: in this study, of the nine symptoms assessed, six were higher in the MRM group (indicating poorer QOL) than in the BCS group. Once again a slight tendency was shown for women treated with conservative surgery to present fewer health problems (symptom scales lower values) and hence a "better" QOL.

The results by surgery type from the QLQ-BR23 questionnaire, designed to measure $\mathrm{QOL}$ in women with $\mathrm{BC}$, were consistent with other studies which also reported that the only significant difference between the two groups (BCS or MRM) was body image. ${ }^{6,12}$

The BCS group showed lower levels on the symptom scale than the MRM group, consequently reporting fewer health problems, with the exception of hair loss. This result is consistent with other studies, in which similar results have been reported. ${ }^{6,12,21}$
In general, women receiving BCS perceive a better QOL than women treated with MRM, with the exception of hair loss, in which both groups showed a similar level of concern.

\section{Study limitations}

Results of the present investigation are limited by the small sample size and by the cross-sectional design. The effects of chemotherapy on QOL were not investigated because both groups used similar drug treatments. No radiotherapy equipment was available at the study site. We acknowledge that future investigations should also address the effect of chemotherapy and/or radiotherapy on QOL.

Our research group is small and independent; no grant or financial support was received to conduct the present investigation. A larger sample with more sociodemographic and clinical variables would need extra economic and human resources. Our results are preliminary and not conclusive.

\section{Conclusion}

In the study sample, MRM and BCS are essentially equivalent choices in terms of QOL with the exception of impact on body image. In general, women who received BCS had a better QOL perception than those treated with MRM, although the differences were not statistically significant.

\section{Disclosure}

The authors report no conflicts of interest in this work.

\section{References}

1. Pandey M, Thomas BC, SreeRekha P, et al. Quality of life determinants in women with breast cancer undergoing treatments with curative intent. World J Surg Oncol. 2005;3:63.

2. Groot MT, Baltussen R, Uyl-de-Groot CA, Andrson BO, Hortoágyi GN Costs and health effects of breast cancer interventions in epidemiologically different regions of Africa, North America, and Asia. Breast J. 2006;12(Supp1 1):S81-S90. 
3. Secretaría de Salud. Situación Actual del Cáncer de Mama en México. C2008. Cited Jan 10, 2008. Available from: http://www.generoysaludreproductiva.gob.mx/.

4. Cancer De Mama. ¿Que es el cancer de mama? 2008. Accessed Jun 20, 2008. Available from: http://www.cancermama.com.mx/QueEsRV/ CancerMama/.

5. Organización Mundial de la Salud. Cómo se encarga el informe sobre la salud en el mundo. 2006. Accessed Oct 15, 2007. Available from: http://www.who.int/whr/order/es/.

6. King MT, Kenny P, Shiell A, Hall J, Boyages J. Quality of life three months and one year after first treatment for early stage breast cancer: Influence of treatment and patient characteristics. Qual Life Res. 2000;9:789-800.

7. Jacobson JA, Danforth DN, Cowan KH, et al. Ten-year results of a comparison of conservation with mastectomy in the treatment of stage I and II breast cancer. N Engl J Med. 1995;332:907-911.

8. Broeckel JA, Jacobsen PB, Balducci L, Horton J, Lyman GH. Quality of life after adjuvant chemotherapy for breast cancer. Breast Cancer Res Treat. 2000;62:141-150.

9. Radice D, Redaelli A. Breast cancer management. Quality of life and cost considerations. Pharmacoeconomics. 2003;21:383-396.

10. Plan Nacional de Salud. Secretaría de Salud del DF. 2004. Accessed Oct 15, 2007. Available from: http://www.salud.gob.mx/.

11. Carlson LE, Bultz BD. Benefits of psychosocial oncology care: Improved quality of life and medical cost offset. Health Qual Life Outcomes. 2003;1:8-17.

12. Yong C, Xiao-Ou S, Yutang G, et al. The Long-term impact of medical and socio-demographic factors on the quality of life of breast cancer survivors among Chinese women. Breast Cancer Res Treat. 2004;87:135-147.
13. Casso D, Buist DSM, Taplin S. Quality of life of 5-10 year breast cancer survivors diagnosed between age 40 and 49. Health Qual Life Outcomes. 2004;2:25-34.

14. Vacek PM, Winstead-Fry P, Secker-Walker RH, Hooper GJ. Factors influencing quality of life in breast cancer survivors. Qual Life Res. 2003;12:527-537.

15. Remennick L. The challenge of early breast cancer detection among immigrant and minority women in multicultural societies. Breast $J$, 2006;12(Suppl 1):S103-10.

16. Aaronson NK, Ahmedzai S, Bergman B, et al. The European Organization for Research and Treatment of Cancer QLQ-C30: a quality-of-life instrument for use in international clinical trials in oncology. $\mathrm{J} \mathrm{Natl}$ Cancer Inst. 1993;85:365-376.

17. Sprangers MAJ, Groenvold M, Arraras JI, et al. The European Organization for Research and Treatment of Cancer breast cancer-specific quality-of-life questionnaire module: first results from a three-country field study. J Clin Oncol. 1996;14:2756-2768.

18. Fayers PM, Aaronson NK, Bjordal K, Corran M, Groenvol M; on behalf of the EORTC Quality of Life Study Group. EORTC QLQ-C30 Scoring Manual. Third edition. Brussels, Belguim: EORTC Quality of Life Study Group; 2001. pp. 1-86.

19. Young T, de Haes H, Curran D, et al; on behalf of the EORTC Quality of Life Study Group. Guidelines for Assessing Quality of Life in EORTC Clinical Trials. Brussels, Belguim: EORTC Quality of life Study Group; 1999. pp. 1-41.

20. King MT. The interpretation of scores from the EORTC quality of life questionnaire QLQ-C30. Qual Life Res. 1996;5:555-567.

21. Janz NK, Mujahid M, Lantz PM, et al. Population-based study of the relationship of treatment and sociodemographics on quality of life for early stage breast cancer. Qual Life Res. 2005;14:1467-1479. 